\title{
Impact of Changes in Maternal Age and Parity Distribution on the Increasing Trends in the Low Birth Weight and Very Low Birth Weight Rates in South Korea, 2005-2015
}

\author{
Yujin $\mathrm{Oh}^{1,2}$, Jisuk Bae ${ }^{1}$ \\ ${ }^{1}$ Department of Preventive Medicine, Daegu Catholic University School of Medicine, Daegu, Korea; ${ }^{2}$ Environmental Health Center, Soonchunhyang \\ University Gumi Hospital, Gumi, Korea
}

Objectives: The aim of this study was to evaluate the impact of shifts in maternal age and parity on the increasing trends in the low birth weight (LBW) and very low birth weight (VLBW) rates from 2005 to 2015 in South Korea.

Methods: Data from 4993041 live births registered with Statistics Korea during the period between 2005 and 2015 were analyzed. Applying a modified standardization method, we partitioned the total increment in the LBW and VLBW rates into (1) the increase in the LBW and VLBW rates due to changes in the maternal age and parity distribution (AP-dis) and (2) the increase due to changes in the age-specific and parity-specific rates (AP-spe) of LBW and VLBW for singleton and multiple births, respectively.

Results: During the study period, the total increment in the LBW and VLBW rates was $1.43 \%$ and $0.25 \%$, respectively. Among singleton births, changes in the AP-dis accounted for 79\% (0.34\%p) and 50\% (0.06\%p) of the total increment in the LBW and VLBW rates, respectively. Meanwhile, among multiple births, changes in the AP-dis did not contribute to the increase in the LBW and VLBW rates, with $100 \%$ of the increase in the LBW (1.00\%p) and VLBW $(0.13 \%$ p) rates being attributed to changes in the AP-spe.

Conclusions: This study demonstrated that shifts in maternal age and parity were prominent contributors to the increase in the LBW and VLBW rates among singleton births between 2005 and 2015 in South Korea.

Key words: Infant, Low birth weight, Very low birth weight, Maternal age, Parity

\section{INTRODUCTION}

Received: October 23, 2018 Accepted: February 14, 2019

Corresponding author: Jisuk Bae, MD, PhD

Department of Preventive Medicine, Daegu Catholic University School of Medicine, 33 Duryugongwon-ro 17-gil, Nam-gu, Daegu 42472,

Korea

E-mail: jialove@cu.ac.kr

This is an Open Access article distributed under the terms of the Creative Commons Attribution Non-Commercial License (http://creativecommons.org/licenses/bync/4.0// which permits unrestricted non-commercial use, distribution, and reproduction in any medium, provided the original work is properly cited.
South Korea (hereafter Korea) has the lowest total fertility rate (TFR) among Organization for Economic Cooperation and Development (OECD) countries, of which the average TFR in 2014 was 1.68 [1]. The TFR of Korea decreased from 4.53 in 1970 to 1.57 in 1990, and further decreased from 1.47 in 2000 to 1.24 in 2015 [1]. The reasons for this decline in the TFR include postponed marriage and childbearing and a decrease in desired family size [1]. The proportion of babies born with a low birth weight (LBW), defined as a birth weight less than 
$2500 \mathrm{~g}$, increased from 4.3\% in 2005 to $5.7 \%$ in 2015 in Korea [1]. The proportion of babies born with a very low birth weight (VLBW), defined as a birth weight less than $1500 \mathrm{~g}$, also increased from $0.4 \%$ in 2005 to $0.7 \%$ in 2015 [1]. During the same period, there was a decline in the mean birth weight from $3.25 \mathrm{~kg}$ to $3.20 \mathrm{~kg}$ [1].

Maternal age at delivery and parity are known to be related to the infant's birth weight. It has been reported that women $<20$ years and $\geq 35$ years of age tend to have a higher LBW rate than do those 20-34 years of age [2-7]. In addition, the LBW rate tends to be higher for first births than for second and third births, and then increases as the birth order increases beyond $4[2,3,8,9]$. Other risk factors for LBW and VLBW include maternal socio-demographic factors (e.g., race, marital status, and education level), lifestyle factors (e.g., smoking), underlying medical conditions (e.g., hypertension, kidney disease, and periodontal disease), and environmental factors (e.g., air pollution) [2,3,10-13].

The increase in the LBW and VLBW rates is a public health concern, as LBW and VLBW are related to an increased risk of infant mortality, impaired neurocognitive development in childhood, and various chronic diseases (e.g., hypertension, diabetes, and cardiovascular diseases) in adulthood [14-17]. In order to reduce the social and economic burden of LBW and VLBW, it is important to identify modifiable factors contributing to the increase in the LBW and VLBW rates. In our previous study using data from the national birth registry [18], we demonstrated that shifts in maternal age and parity were important contributors to the increasing trends in the LBW rate among singleton births from 1995 to 2005 in Korea. As a follow-up study [19], we aimed to (1) evaluate the impact of shifts in maternal age and parity on the increasing trends in the LBW and

Table 1. All live births, maternal age and parity distribution, singleton and multiple birth rates, and LBW and VLBW rates from 2005 to 2015 in Korea

\begin{tabular}{|c|c|c|c|c|c|c|c|c|c|c|c|}
\hline & \multicolumn{11}{|c|}{ Year } \\
\hline & 2005 & 2006 & 2007 & 2008 & 2009 & 2010 & 2011 & 2012 & 2013 & 2014 & 2015 \\
\hline All live births $(n)^{1}$ & 430523 & 443552 & 488854 & 461933 & 441692 & 466772 & 469838 & 483256 & 435494 & 434177 & 436950 \\
\hline First & 51.69 & 52.00 & 53.48 & 52.32 & 52.05 & 50.36 & 50.95 & 51.48 & 51.60 & 51.88 & 52.29 \\
\hline Second & 38.70 & 38.52 & 37.15 & 38.09 & 38.48 & 38.95 & 38.07 & 38.06 & 38.02 & 38.06 & 38.00 \\
\hline$\leq 19$ & 0.50 & 0.53 & 0.57 & 0.49 & 0.52 & 0.52 & 0.60 & 0.56 & 0.63 & 0.58 & 0.50 \\
\hline $20-24$ & 7.43 & 6.75 & 6.39 & 5.94 & 5.49 & 5.15 & 5.21 & 5.05 & 5.05 & 4.86 & 4.67 \\
\hline $25-29$ & 40.41 & 38.77 & 38.06 & 36.39 & 35.17 & 31.41 & 29.11 & 26.27 & 23.57 & 22.11 & 21.60 \\
\hline $30-34$ & 41.01 & 42.14 & 41.91 & 42.86 & 43.39 & 45.84 & 47.06 & 49.43 & 50.53 & 50.84 & 49.37 \\
\hline \multicolumn{12}{|l|}{ LBW $_{\text {rate }}^{2}$} \\
\hline All live births & 4.28 & 4.38 & 4.67 & 4.87 & 4.88 & 4.91 & 5.19 & 5.31 & 5.52 & 5.68 & 5.71 \\
\hline Singleton births & 3.25 & 3.29 & 3.31 & 3.50 & 3.49 & 3.51 & 3.68 & 3.67 & 3.77 & 3.83 & 3.75 \\
\hline Multiple births & 50.29 & 48.18 & 52.72 & 53.25 & 54.47 & 54.53 & 55.36 & 54.36 & 56.75 & 56.89 & 57.11 \\
\hline \multicolumn{12}{|l|}{ VLBW rate $^{2}$} \\
\hline All live births & 0.41 & 0.42 & 0.45 & 0.48 & 0.51 & 0.51 & 0.59 & 0.60 & 0.65 & 0.63 & 0.66 \\
\hline Singleton births & 0.33 & 0.32 & 0.33 & 0.36 & 0.36 & 0.37 & 0.43 & 0.43 & 0.45 & 0.44 & 0.45 \\
\hline Multiple births & 4.18 & 4.49 & 4.67 & 4.62 & 5.63 & 5.33 & 5.95 & 5.73 & 6.57 & 5.90 & 6.04 \\
\hline
\end{tabular}

Values are presented as \%.

LBW, low birth weight; VLBW, very low birth weight.

'Live births with a birth weight $<500 \mathrm{~g}(\mathrm{n}=619)$ or missing data for birth weight $(\mathrm{n}=15128)$, birth plurality $(\mathrm{n}=11079)$, maternal age $(\mathrm{n}=2829)$, or parity $(\mathrm{n}=714)$ were excluded from the analysis.

${ }^{2} p_{\text {trend }}<0.001$ calculated by the linear-by-linear association test. 
VLBW rates from 2005 to 2015 in Korea; and (2) compare the results of the current study with those of the previous study.

\section{METHODS}

\section{Research Data}

All live births registered with Statistics Korea during the period between 2005 and 2015 were obtained from the Microdata Integrated Service (MDIS) [1]. Of 5023410 registered births, our analysis was restricted to 4993041 births, after excluding 30369 births with a birth weight $<500 \mathrm{~g}(\mathrm{n}=619)$ or missing data for birth weight $(n=15128)$, birth plurality $(n=11079)$, maternal age $(n=2829)$, or parity $(n=714)$. In consideration of the potential inaccuracy of the gestational age data recorded on birth certificates, our analysis was primarily focused on the birth weight data, with the gestational age data being used for further subgroup analysis.

\section{Data Analysis}

The rates of singleton births, multiple births, LBW births, and VLBW births were estimated for each year from 2005 to 2015. The significance of trends in these rates during the study period was tested by the linear-by-linear association test. Differences in the age-specific and parity-specific rates (AP-spe) of LBW and VLBW between 2005 and 2015 were tested by the chi-square test or the Fisher exact test. To assess statistical significance, a 2-sided significance level of 0.05 was used. Statistical analysis was performed using SPSS version 19 (IBM Corp., Armonk, NY, USA).

A modified standardization method, as previously described in detail [18], was used to partition the increase in the LBW and VLBW rates between 2005 and 2015 into the following 2 components: (1) the increase due to changes in the maternal age and parity distribution (AP-dis); and (2) the increase due to changes in the AP-spe. Briefly, when the standard population in 2005 is cross-tabulated by age, $i=1,2, \ldots, i(\leq 19,20$ $24,25-29,30-34,35-39$, and $\geq 40)$ and parity, $j=1,2, \ldots, j(1,2$, and $\geq 3$ ), the LBW (or VLBW) rate in the ij-th cell, $\mathrm{m}_{\mathrm{ij}}^{*}$ (where the asterisk denotes the standard population), is

$$
m_{i j}^{*}=\frac{l_{i j}^{*}}{b_{i j}^{*}}
$$

where $\mathrm{l}_{\mathrm{ij}}$ is the number of LBW (or VLBW) births in the $\mathrm{ij}$-th cell, and $b_{i j}$ is the number of births in the ij-th cell. The overall LBW (or VLBW) rate, $\mathrm{M}^{*}$ is

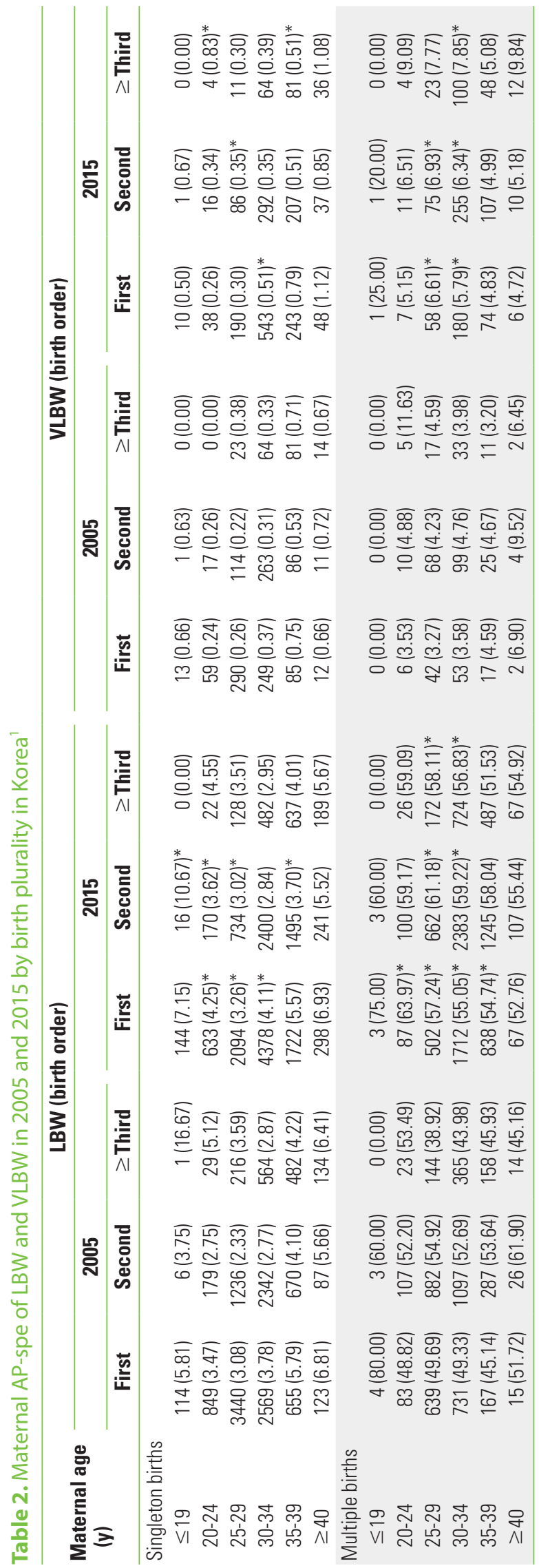

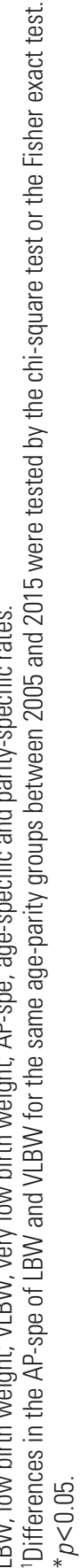




$$
\mathrm{M}^{*}=\frac{\mathrm{L}^{*}}{\mathrm{~B}^{*}}=\sum_{\mathrm{ij}} \mathrm{m}_{\mathrm{ij}}^{*} \mathrm{~b}_{\mathrm{ij}}^{*}
$$

where $L$ is the total number of LBW (or VLBW) births, and $B$ is the total number of births. The ratio of $m_{i j}^{*}$ to $M^{*}, k_{i j}^{*}$, is

$$
\mathrm{k}_{\mathrm{ij}}^{*}=\frac{\mathrm{m}_{\mathrm{ij}}^{*}}{\mathrm{M}^{*}}=\frac{\frac{\mathrm{l}_{\mathrm{ij}}^{*}}{\mathrm{~b}_{\mathrm{ij}}^{*}}}{\frac{\mathrm{L}^{*}}{\mathrm{~B}^{*}}}
$$

The age-parity score, $\mathrm{K}$ is

$\mathrm{K}=\sum_{\mathrm{ij}} \mathrm{k}_{\mathrm{ij}} \mathrm{b}_{\mathrm{ij}}=\sum_{\mathrm{ij}} \frac{\mathrm{m}^{*}{ }_{\mathrm{ij}} \mathrm{b}_{\mathrm{ij}}}{\mathrm{M}^{*}}=\frac{\sum_{\mathrm{ij}} \mathrm{m}^{*} \mathrm{~b}_{\mathrm{ij}}}{\mathrm{M}^{*}}=\frac{\text { Expected LBW (or VLBW) rate in } 2015}{\text { Observed LBW (or VLBW) rate in } 2005}$ where $\frac{b_{i j}}{B}$ is the proportion of births in 2015 in the ij-th cell. $\mathrm{K}$ is directly interpreted as the percentage difference in the LBW (or VLBW) rate with reference to the standard population in 2005. The 2 components of the total increment of the LBW (or VLBW) rate were calculated as follows: (1) the increase due to changes in the AP-dis = expected LBW (or VLBW) rate in 2015 - observed LBW (or VLBW) rate in 2005; and (2) the increase due to changes in the AP-spe $=$ observed LBW (or VLBW) rate in 2015 - expected LBW (or VLBW) rate in 2015. We calculated these 2 components for singleton and multiple births, respectively. As all of the information used was deidentified, this study was classified as exempt research by the Institutional Review Board of Daegu Catholic University Medical Center.

\section{RESULTS}

Between 2005 and 2015, the singleton birth rate steadily decreased from $97.81 \%$ in 2005 to $96.32 \%$ in 2015 , whereas the multiple birth rate gradually increased from $2.19 \%$ in 2005 to $3.68 \%$ in 2015 . During the same period, statistically signifi- cant increasing trends in the LBW and VLBW rates were observed among both singleton and multiple births ( $p_{\text {trend }}<$ 0.001 ). Among all live births, the LBW rate increased from $4.28 \%$ in 2005 to $5.71 \%$ in 2015 ; and the VLBW rate increased from $0.41 \%$ in 2005 to $0.66 \%$ in 2015 . The LBW and VLBW rates for singleton births increased from $3.25 \%$ and $0.33 \%$ in 2005 to $3.75 \%$ and $0.45 \%$ in 2015 , respectively. The LBW and VLBW rates for multiple births increased from $50.29 \%$ and $4.18 \%$ in 2005 to $57.11 \%$ and $6.04 \%$ in 2015 , respectively. Of note, the LBW and VLBW rates for multiple births were approximately 15 times and 13 times higher than those for singleton births, respectively (Table 1).

Table 2 shows the AP-spe of LBW and VLBW in 2005 and 2015 by birth plurality. Among singleton births, women $<20$ years and $\geq 35$ years of age had higher LBW rates than did those 20-34 years of age in both years, irrespective of birth order. Meanwhile, no such feature was observed among multiple births. When the AP-spe of LBW in 2015 were compared with those in 2005 among singleton births, the rates in 2015 were significantly higher than those in 2005 in 6 out of 18 ageparity groups $(p<0.05)$. Among multiple births, 8 out of 18 age-parity groups showed a statistically significant increase in the rates between 2005 and $2015(p<0.05)$. A statistically significant increase in the AP-spe of VLBW between 2005 and 2015 was noted in 3 out of 18 age-parity groups among singleton births and 5 out of 18 age-parity groups among multiple births $(p<0.05)$ (Table 2).

Table 3 describes the calculation process for partitioning the total increment in the LBW rate into its 2 components: (1) the increase due to changes in the AP-dis, and (2) the increase due to changes in the AP-spe by birth plurality. The absolute percentage of the total increment in the LBW rate between 2005 and 2015 was $1.43 \%$ p (LBW rate: $4.28 \%$ in 2005 vs. $5.71 \%$ in 2015), of which $0.43 \%$ p occurred among singleton births and

Table 3. Process of calculating changes in the LBW rate due to changes in the AP-dis and changes in the AP-spe among single-

\begin{tabular}{|c|c|c|c|c|c|c|}
\hline \multirow{2}{*}{ Variables } & \multicolumn{2}{|c|}{ All live births } & \multicolumn{2}{|c|}{ Singleton births } & \multicolumn{2}{|c|}{ Multiple births } \\
\hline & 2005 & 2015 & 2005 & 2015 & 2005 & 2015 \\
\hline Observed absolute \%p of LBW rate & 4.28 & 5.71 & $3.18^{\mathrm{a}}$ & $3.61^{\mathrm{b}}$ & $1.10^{\mathrm{c}}$ & $2.10^{\mathrm{d}}$ \\
\hline Changes in the LBW rate (\%p) & \multicolumn{2}{|c|}{$1.43(e+f)$} & \multicolumn{2}{|c|}{$0.43^{e}(b-a)$} & \multicolumn{2}{|c|}{$1.00^{f}(d-c)$} \\
\hline Age-parity score for 2015 & & & \multicolumn{2}{|c|}{$1.108^{g}$} & \multicolumn{2}{|c|}{$0.996^{h}$} \\
\hline Expected absolute \%p of LBW rate in 2015 when there were no changes in the AP-spe & & & \multicolumn{2}{|c|}{$3.53^{\mathrm{i}}(\mathrm{a} \times \mathrm{g})$} & \multicolumn{2}{|c|}{$1.10^{\mathrm{i}}(\mathrm{c} \times \mathrm{h})$} \\
\hline Absolute \%p of increase in the LBW rate due to changes in the AP-dis & \multicolumn{2}{|c|}{$0.34(k+1)$} & \multicolumn{2}{|c|}{$0.34^{k}(i-a)$} & \multicolumn{2}{|c|}{$0.00^{\prime}(j-c)$} \\
\hline Absolute \%p of increase in the LBW rate due to changes in the AP-spe & \multicolumn{2}{|c|}{$1.09(m+n)$} & \multicolumn{2}{|c|}{$0.09^{m}(b-i)$} & \multicolumn{2}{|c|}{$1.00^{n}(d-j)$} \\
\hline
\end{tabular}
ton and multiple births between 2005 and 2015 in Korea

LBW, low birth weight; AP-dis, age and parity distribution; AP-spe, age-specific and parity-specific rates. 
Table 4. Process of calculating changes in the VLBW rate due to changes in the AP-dis and changes in the AP-spe among singleton and multiple births between 2005 and 2015 in Korea

\begin{tabular}{|c|c|c|c|c|c|c|}
\hline \multirow{2}{*}{ Variables } & \multicolumn{2}{|c|}{ All live births } & \multicolumn{2}{|c|}{ Singleton births } & \multicolumn{2}{|c|}{ Multiple births } \\
\hline & 2005 & 2015 & 2005 & 2015 & 2005 & 2015 \\
\hline Observed absolute \%p of VLBW rate & 0.41 & 0.66 & $0.32^{\mathrm{a}}$ & $0.44^{\mathrm{b}}$ & $0.09^{c}$ & $0.22^{\mathrm{d}}$ \\
\hline Changes in the VLBW rate (\%p) & \multicolumn{2}{|c|}{$0.25(e+f)$} & \multicolumn{2}{|c|}{$0.12^{\mathrm{e}}(\mathrm{b}-\mathrm{a})$} & \multicolumn{2}{|c|}{$0.13^{f}(d-c)$} \\
\hline Age-parity score for 2015 & & & \multicolumn{2}{|c|}{$1.188^{9}$} & \multicolumn{2}{|c|}{$1.035^{\mathrm{h}}$} \\
\hline Expected absolute \%p of VLBW rate in 2015 when there were no changes in the AP-spe & & & \multicolumn{2}{|c|}{$0.38^{i}(a \times g)$} & \multicolumn{2}{|c|}{$0.09^{j}(c \times h)$} \\
\hline Absolute \%p of increase in the VLBW rate due to changes in the AP-dis & \multicolumn{2}{|c|}{$0.06(k+1)$} & \multicolumn{2}{|c|}{$0.06^{k}(i-a)$} & \multicolumn{2}{|c|}{$0.00^{\prime}(j-c)$} \\
\hline Absolute \%p of increase in the VLBW rate due to changes in the AP-spe & \multicolumn{2}{|c|}{$0.18(m+n)$} & \multicolumn{2}{|c|}{$0.06^{m}(b-i)$} & \multicolumn{2}{|c|}{$0.13^{n}(d-j)$} \\
\hline
\end{tabular}

VLBW, very low birth weight; AP-dis, age and parity distribution; AP-spe, age-specific and parity-specific rates.

1.00\%p occurred among multiple births. Among singleton births, changes in the AP-dis accounted for $79 \%$ (0.34\%p) of the total increase in the LBW rate. Among multiple births, in contrast, changes in the AP-dis did not contribute to the increase in the LBW rate, with $100 \%$ (1.00\%p) of the increase in the LBW rate being attributed to changes in the AP-spe (Table 3). As presented in Table 4, the absolute percentage of the total increment in the VLBW rate between 2005 and 2015 was $0.25 \%$ points (VLBW rate: $0.41 \%$ in 2005 vs. $0.66 \%$ in 2015 ), of which $0.12 \%$ p occurred among singleton births and $0.13 \% p$ occurred among multiple births. Among singleton births, changes in the AP-dis accounted for $50 \%(0.06 \% \mathrm{p})$ of the total increase in the VLBW rate. However, among multiple births, $100 \%(0.13 \%$ p) of the increase in the VLBW rate was attributed to changes in the AP-spe (Table 4).

\section{DISCUSSION}

In this study using data from the national birth registry, we evaluated the impact of shifts in maternal age and parity on the increasing trends in the LBW and VLBW rates from 2005 to 2015 in Korea. The current study demonstrated that changes in the AP-dis accounted for $79 \%(0.34 \% \mathrm{p})$ of the total increase in the LBW rate and $50 \%(0.06 \%$ p) of the total increase in the VLBW rate among singleton births. Among multiple births, however, $100 \%$ of the total increase in the LBW $(1.00 \%$ p) and VLBW $(0.13 \%$ p) rates was attributed to changes in the AP-spe. In our previous study [18] of 6397945 live births registered with Statistics Korea during the period between 1995 and 2005 , changes in the AP-dis were found to account for $50 \%$ $(0.32 \% p)$ of the total increase in the LBW rate among singleton births. When comparing our previous results with the current findings, the absolute percentage of the increase in the
LBW rate due to changes in the AP-dis slightly increased from 0.32\%p (between 1995 and 2005) to 0.34\%p (between 2005 and 2015). Meanwhile, among multiple births, both our previous and current findings indicated that changes in the AP-spe accounted for $100 \%$ of the total increase in the LBW rate (0.62\%p between 1995 and 2005; 1.00\%p between 2005 and 2015).

In a study conducted in the USA [20], the impact of shifts in maternal age and parity on the LBW and VLBW rates from 1980 and 2000 was investigated by race/ethnicity. That study showed that during the period between 1980 and 1990, changes in the AP-dis accounted for $32.5 \%$ and $15.9 \%$ of the total increase in the VLBW rate among whites and blacks, respectively. In addition, during the period between 1990 and 2000, changes in the AP-dis accounted for $6.3 \%, 9.9 \%$, and $16.8 \%$ of the total increase in the VLBW among non-Hispanic whites, Hispanics, and blacks, respectively. In regard to the LBW rate, increasing trends in the rate were noted only for blacks from 1980 to 1990 , indicating that $38.2 \%$ of the total increase could be attributed to changes in the AP-dis. When comparing those findings with the findings from the current study, the contribution of shifts in maternal age and parity to the increase in the LBW and VLBW rates was much smaller among the USA population than among the Korean population. This may be partly explained by racial differences in the etiology of LBW and VLBW or the degree of shifts in maternal age and parity [18].

Indeed, the current study demonstrated that the proportion of live births to mothers aged 35-39 years increased dramatically from $9.37 \%$ in 2005 to $21.01 \%$ in 2015 (Table 1). The proportion of live births to mothers aged $\geq 40$ years likewise increased from $1.29 \%$ in 2005 to $2.85 \%$ in 2015 (Table 1). Such changes in the maternal age distribution may be related to a variety of social factors, such as delayed marriage and child- 
bearing, increased female economic participation, and changes in obstetric practices including the increased use of assisted reproductive technology (ART) $[1,21,22]$. In addition, the current study revealed that the multiple birth rate increased from $2.19 \%$ to $3.68 \%$ during the same period (Table 1). It is worth noting that $1.00 \% \mathrm{p}(69.9 \%)$ of the $1.43 \% \mathrm{p}$ total increase in the LBW rate and $0.13 \%$ p $(52.0 \%)$ of the $0.25 \%$ p total increase in the VLBW rate occurred among multiple births (Tables 3 and 4). The use of ART, especially the number of embryos transferred, is known to be associated with an increased multiple birth rate $[21,22]$. In consideration of the lack of apparent changes in the maternal parity distribution during the period between 2005 and 2015 (Table 1), we further evaluated the sole effect of shifts in maternal age on the increasing trends in the LBW and VLBW rates during the study period. The results indicated that changes in the maternal age distribution accounted for $60 \%(0.26 \% \mathrm{p}$; $<79 \%$ when evaluating the combined effect of shifts in maternal age and parity) of the total increase in the LBW rate and $50 \%(0.06 \%$ p) of the total increase in the VLBW rate among singleton births, reflecting that the sole effect of shifts in maternal parity on the VLBW rate was almost negligible (data not shown).

We utilized the birth weight data from the national birth registry, as those data are considered to be more accurate than the gestational age data recorded on birth certificates. Nevertheless, it is noteworthy that LBW and VLBW represent a mixture of both preterm birth and intrauterine growth restriction from an etiological perspective [23]. Namely, preterm birth, which comprises deliveries at less than 37 completed weeks of gestation, is causally related to LBW and VLBW. A more stringent criterion of less than 34 completed weeks of gestation is frequently utilized in clinical settings, as deliveries of this duration of gestation have been reported to be associated with perinatal complications, including respiratory distress syndrome $[24,25]$. When our analysis was restricted to deliveries at 34 or more completed weeks of gestation, changes in the AP-dis accounted for $88 \%$ (0.23\%p; > 79\% when analyzed without regard to gestational age) of the total increase in the LBW rate among singleton births $(n=855$ 195; 1177 deliveries of unknown gestational age were excluded from the analysis). This suggests that shifts in maternal age and parity may have had a greater impact on the increase in the LBW rate among growth-restricted term infants than among preterm infants.

The major limitations of the current study should be taken into account, when interpreting the study results. One potential limitation is the possibility of inaccuracies in the birth weight data recorded on birth certificates, which could have led to misclassification of LBW and VLBW. Furthermore, we were unable to investigate the reasons for changes in the APspe in our dataset, as the birth registry data provided limited information about the potential etiologic factors for LBW and VLBW of parents and infants. Plausible factors associated with the increase in the LBW and VLBW rates may include lifestyle factors such as cigarette smoking [26] and environmental factors such as air pollution $[27,28]$. Likewise, an in-depth analysis considering other related factors, such as obstetric practices (e.g., ART) and maternal complications (e.g., gestational hypertension, gestational diabetes) was not possible due to the limited information in our dataset. Further comprehensive research is needed to identify various modifiable and environmental factors contributing to the increase in the LBW and VLBW rates.

In conclusion, this study demonstrated that shifts in maternal age and parity were prominent contributors to the increase in the LBW and VLBW rates among singleton births between 2005 and 2015 in Korea. The increased multiple birth rate may partly explain the increase in the LBW and VLBW rates during the same period, although the increase in the LBW and VLBW rates among multiple births were not found to be explained by shifts in maternal age and parity. In light of current social trends regarding delayed marriage and childbearing, efforts should be made to provide women of childbearing age with reproductive health services and social interventions aiming to facilitate pregnancy at a physiologically optimal age and to reduce adverse birth outcomes, including LBW and VLBW.

\section{CONFLICT OF INTEREST}

The authors have no conflicts of interest associated with the material presented in this paper.

\section{ACKNOWLEDGEMENTS}

This article is based on the first author's dissertation submitted to Daegu Catholic University in 2018.

This work was supported by research grants from Daegu Catholic University in 2017. 


\section{ORCID}

Yujin Oh http://orcid.org/0000-0002-8669-1361

Jisuk Bae http://orcid.org/0000-0002-5809-5394

\section{REFERENCES}

1. Statistics Korea. Annual report on live births and deaths statistics, 2005-2015 [cited 2018 Oct 23]. Available from: http:// kosis.kr/publication/publicationThema.do (Korean).

2. Deshmukh JS, Motghare DD, Zodpey SP, Wadhva SK. Low birth weight and associated maternal factors in an urban area. Indian Pediatr 1998;35(1):33-36.

3. Valero De Bernabé J, Soriano T, Albaladejo R, Juarranz M, Calle ME, Martínez D, et al. Risk factors for low birth weight: a review. Eur J Obstet Gynecol Reprod Biol 2004;116(1):3-15.

4. Carolan M, Frankowska D. Advanced maternal age and adverse perinatal outcome: a review of the evidence. Midwifery 2011;27(6):793-801.

5. Roth J, Hendrickson J, Schilling M, Stowell DW. The risk of teen mothers having low birth weight babies: implications of recent medical research for school health personnel. J Sch Health 1998;68(7):271-275.

6. Salem Yaniv S, Levy A, Wiznitzer A, Holcberg G, Mazor M, Sheiner E. A significant linear association exists between advanced maternal age and adverse perinatal outcome. Arch Gynecol Obstet 2011;283(4):755-759.

7. Ziadeh S. Obstetric outcome of teenage pregnancies in North Jordan. Arch Gynecol Obstet 2001;265(1):26-29.

8. Shah PS; Knowledge Synthesis Group on Determinants of LBW/ PT births. Parity and low birth weight and preterm birth: a systematic review and meta-analyses. Acta Obstet Gynecol Scand 2010;89(7):862-875.

9. Silva AA, Barbieri MA, Gomes UA, Bettiol H. Trends in low birth weight: a comparison of two birth cohorts separated by a 15year interval in Ribeirão Preto, Brazil. Bull World Health Organ 1998;76(1):73-84.

10. Balte P, Karmaus W, Roberts G, Kurukulaaratchy R, Mitchell F, Arshad H. Relationship between birth weight, maternal smoking during pregnancy and childhood and adolescent lung function: a path analysis. Respir Med 2016;121:13-20.

11. Bosetti C, Nieuwenhuijsen MJ, Gallus S, Cipriani S, La Vecchia C, Parazzini F. Ambient particulate matter and preterm birth or birth weight: a review of the literature. Arch Toxicol 2010;84(6): 447-460.
12. Han Z, Mulla S, Beyene J, Liao G, McDonald SD; Knowledge Synthesis Group. Maternal underweight and the risk of preterm birth and low birth weight: a systematic review and meta-analyses. Int J Epidemiol 2011;40(1):65-101.

13. Teshome A, Yitayeh A. Relationship between periodontal disease and preterm low birth weight: systematic review. Pan Afr Med J 2016;24:215.

14. ljzerman RG, Boomsma DI, Stehouwer CD. Intrauterine environmental and genetic influences on the association between birthweight and cardiovascular risk factors: studies in twins as a means of testing the fetal origins hypothesis. Paediatr Perinat Epidemiol 2005;19 Suppl 1:10-14.

15. McCormick MC. Significance of low birthweight for infant mortality and morbidity. Birth Defects Orig Artic Ser 1988;24(1):3-10.

16. McNeill G, Tuya C, Smith WC. The role of genetic and environmental factors in the association between birthweight and blood pressure: evidence from meta-analysis of twin studies. Int J Epidemiol 2004;33(5):995-1001.

17. Osmond C, Barker DJ. Fetal, infant, and childhood growth are predictors of coronary heart disease, diabetes, and hypertension in adult men and women. Environ Health Perspect 2000; 108 Suppl 3:545-553.

18. Bae J, Park JH, Park YK, Kim JY, Lee SW, Park SW. Changes in the distribution of maternal age and parity and increasing trends in the low birth weight rate in Korea between 1995 and 2005. J Prev Med Public Health 2011;44(3):111-117.

19. Oh Y. Impact of changes in maternal age and parity distribution on the increasing trend in the low birth weight and very low birth weight rates in Korea, 2005-2015 [dissertation]. Daegu: Daegu Catholic University; 2018 (Korean).

20. Yang Q, Greenland S, Flanders WD. Associations of maternal age- and parity-related factors with trends in low-birthweight rates: United States, 1980 through 2000. Am J Public Health 2006;96(5):856-861.

21. Centers for Disease Control and Prevention. 2015 Assisted reproductive technology fertility clinic success rates report; 2017 [cited 2018 Oct 23]. Available from: https://www.cdc.gov/art/ reports/2015/fertility-clinic.html.

22. Korean Institute for Health and Society Affairs. 2014 Results analysis and assessment of ART to pregnancy. Sejong: Ministry of Health Welfare; 2015, p. 17-21 (Korean).

23. Louis GM, Platt RW. Reproductive and perinatal epidemiology. New York: Oxford University Press; 2011, p. 152-185.

24. Arnon S, Dolfin T, Litmanovitz I, Regev R, Bauer S, Fejgin M. Preterm labour at 34-36 weeks of gestation: should it be ar- 
rested? Paediatr Perinat Epidemiol 2001;15(3):252-256.

25. Jones SC, Brost BC, Brehm WT. Should intravenous tocolysis be considered beyond 34 weeks' gestation? Am J Obstet Gynecol 2000;183(2):356-360.

26. Elkin ER, O'Neill MS. Trends in environmental tobacco smoke (ETS) exposure and preterm birth: use of smoking bans and direct ETS exposure assessments in study designs. Chem Res Toxicol 2017;30(7):1376-1383.

27. Wilhelm M, Ghosh JK, Su J, Cockburn M, Jerrett M, Ritz B. Traf- fic-related air toxics and preterm birth: a population-based case-control study in Los Angeles County, California. Environ Health 2011;10:89.

28. Kingsley SL, Eliot MN, Glazer K, Awad YA, Schwartz JD, Savitz $D A$, et al. Maternal ambient air pollution, preterm birth and markers of fetal growth in Rhode Island: results of a hospitalbased linkage study. J Epidemiol Community Health 2017; 71(12):1131-1136. 